\title{
Quantitative analysis of cementitious materials by X-ray ptychographic nanotomography
}

\author{
S. Shirani, ${ }^{1}$ A. Cuesta, ${ }^{1}$ A.G. De la Torre, ${ }^{1}$ J.C. da Silva, ${ }^{2}$ D. Karpov, ${ }^{3}$ A. Diaz, ${ }^{4}$ M. Holler, ${ }^{4}$ P. Trtik, ${ }^{4,5}$ \\ M.A.G. Aranda, ${ }^{1}$
}

\begin{abstract}
${ }^{1}$ Departamento de Química Inorgánica, Universidad de Malaga, 29071-Málaga (Spain), ${ }^{2}$ Department Physics Light Matter, Univ. Grenoble Alpes, CNRS, Grenoble INP, Institut NEEL - 38000 Grenoble (France), ${ }^{3}$ European Synchrotron Radiation Facility (ESRF), BP 220-38043 Grenoble (France) ${ }^{4}$ Paul Scherrer Institut, 5232 Villigen PSI (Switzerland), ${ }_{5}^{5}$ Faculty of Civil Engineering, Czech Technical University in Prague, 16629 Prague (Czech Republic),

\section{g_aranda@uma.es}

Cement manufacturing is responsible for $\sim 7 \%$ of the anthropogenic $\mathrm{CO}_{2}$ emissions and hence, decreasing the $\mathrm{CO}_{2}$ footprint, in a sustainable, safe, and cost-effective way, is a top priority. It is also key to develop more durable binders as the estimated world concrete stock is $315 \mathrm{Gt}$ which currently results in $\sim 0.3 \mathrm{Gt} / \mathrm{yr}$ of concrete demolition waste (CDW). Moreover, models under development predict a skyrocketing increase of CDW to $20-40 \mathrm{Gt} / \mathrm{yr}$ by 2100 . This amount could not be easily reprocessed as aggregates for new concretes as such volumes would be more than two times the predicted need. Furthermore, concretes have very complex hierarchical microstructures. The largest components are coarse aggregates with dimensions bigger than a few centimetres and the smallest ones are amorphous components and the calcium silicate hydrate gel with nanoparticle sizes smaller than a few nanometres. To fully understand the properties of current and new cement binders and to optimize their performances, a sound description of their spatially resolved contents is compulsory. However, there is not a tomographic technique that can cover the spatial range of heterogeneity and features of concretes and mortars. This can only be attained within a multitechnique approach overlapping the spatial scales in order to build an accurate picture of the different microstructural features. Here, we have employed far-field and near-field synchrotron X-ray ptychographic nanotomographies to gain a deeper insight into the submicrometer microstructures of Portland cement binders. With these techniques, the available fields of view range from 40 to $300 \mu \mathrm{m}$ with a true spatial resolution (not voxel sizes) evolving between $\sim 50 \mathrm{~nm}$ to $\sim 300 \mathrm{~nm}$. It is explicitly acknowledged here that other techniques like X-ray synchrotron microtomography are necessary to develop the whole picture accessing to larger fields of view (millimetres and even centimetres) albeit with poorer spatial resolution and without the quantitativeness in the reconstructed electron densities.

After framing the problems which are being tackled, we plan to present here our recent results using X-ray ptychographic nanotomography. We will start introducing the outputs obtained using far-field ptychographic nanotomography to determine phase assemblages and mass densities of amorphous components [1,2]. Then, we will move to cover the secondary porosity induced by cement conversion with temperature [3]. Finally, we will present our ongoing work with near-field ptychographic nanotomography in Portland and Belite cements with a larger field of view, capillaries from 200 to $300 \mu \mathrm{m}$ of diameter. Between other features, Hadley grains (hollow shells hydrated particles) have been imaged in 3D and their properties are being statistically extracted, see Figure $\mathbf{1}$. Further details will be discussed and the comparison between far-field and near-field nanotomographies will be carried out.

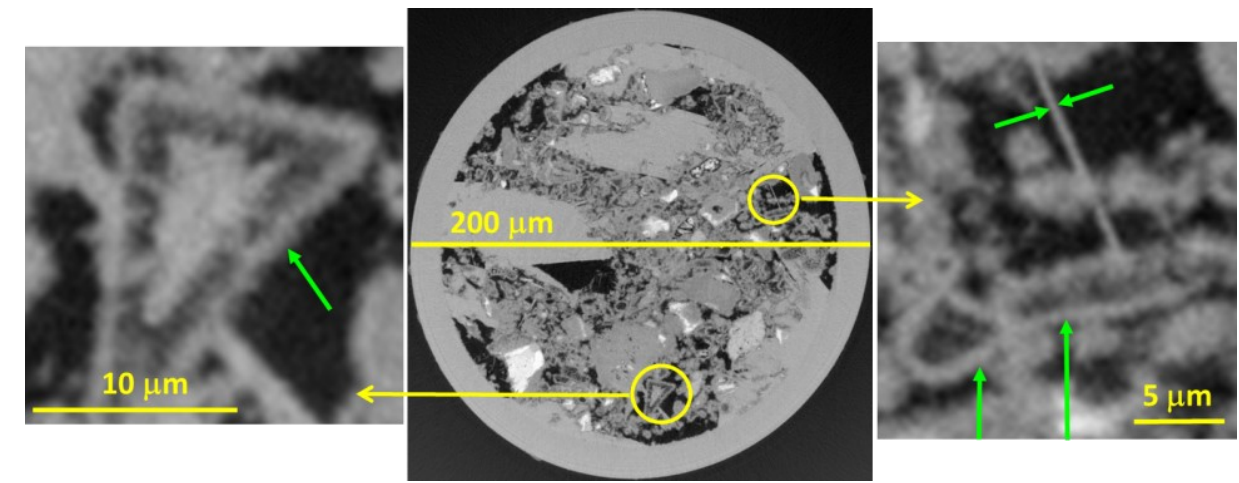

Figure 1. (Centre) orthoslice of the Portland paste capillary reconstructed by near-field ptychographic nanotomography. (Left) Enlarged view of a large Hadley grain typical of the alite hydration mechanism. (Right) $500 \mathrm{~nm}$ thick ettringite needle highlighting the excellent spatial resolution for such a large field of view (two other smaller Hadley grains are evident here).

[1] Cuesta, A., et al. (2017) Chemistry and Mass Density of Aluminum Hydroxide Gel in Eco-Cements by Ptychographic X-ray Computed Tomography. J. Phys. Chem. C, 121, 3044-3054.

[2] Cuesta, A., et al. (2019) Quantitative disentanglement of nanocrystalline phases in cement pastes by synchrotron ptychographic X-ray tomography. IUCrJ, 6, 473-491.

[3] Shirani, S., et al. (2020) Calcium aluminate cement conversion analysed by ptychographic nanotomography. Cem. Con. Res. 137, 106201.

Keywords: synchrotron tomography, Rietveld analysis, ptychography, amorphous components, mass density Acta Cryst. (2021), A77, C183 